University of Nebraska - Lincoln

DigitalCommons@University of Nebraska - Lincoln

September 2007

\title{
Quantum confinement of crystalline silicon nanotubes with nonuniform wall thickness: Implication to modulation doping
}

\author{
Binghai Yan \\ Tsinghua University \\ Xiao Cheng Zeng \\ University of Nebraska-Lincoln, xzeng1@unl.edu \\ Jian $\mathrm{Wu}$ \\ Tsinghua University \\ Bing-Lin Gu \\ Tsinghua University, Beijing 100084, People's Republic of China \\ Wenghui Duan \\ Tsinghua University, Beijing 100084, People's Republic of China
}

Follow this and additional works at: https://digitalcommons.unl.edu/chemzeng

Part of the Chemistry Commons

Yan, Binghai; Zeng, Xiao Cheng; Wu, Jian; Gu, Bing-Lin; and Duan, Wenghui, "Quantum confinement of crystalline silicon nanotubes with nonuniform wall thickness: Implication to modulation doping" (2007). Xiao Cheng Zeng Publications. 79.

https://digitalcommons.unl.edu/chemzeng/79

This Article is brought to you for free and open access by the Published Research - Department of Chemistry at DigitalCommons@University of Nebraska - Lincoln. It has been accepted for inclusion in Xiao Cheng Zeng Publications by an authorized administrator of DigitalCommons@University of Nebraska - Lincoln. 


\title{
Quantum confinement of crystalline silicon nanotubes with nonuniform wall thickness: Implication to modulation doping
}

\author{
Binghai Yan and Gang Zhou \\ Center for Advanced Study, Tsinghua University, Beijing 100084, People's Republic of China \\ and Department of Physics, Tsinghua University, Beijing 100084, People's Republic of China \\ Xiao Cheng Zeng \\ Department of Chemistry, University of Nebraska, Lincoln, Nebraska 68588 \\ and Department of Physics, University of Nebraska, Lincoln, Nebraska 68588 \\ Jian Wu, Bing-Lin Gu, and Wenhui Duan ${ }^{\mathrm{a})}$ \\ Department of Physics, Tsinghua University, Beijing 100084, People's Republic of China
}

(Received 23 May 2007; accepted 12 August 2007; published online 4 September 2007)

\begin{abstract}
First-principles calculations of crystalline silicon nanotubes (SiNTs) show that nonuniformity in wall thickness can cause sizable variation in the band gap as well as notable shift in the optical absorption spectrum. A unique quantum confinement behavior is observed: the electronic wave functions of the valence band maximum and conduction band minimum are due mainly to atoms located in the thicker side of the tube wall. This is advantageous to spatially separate the doping impurities from the conducting channel in doped SiNTs. Practically, the performance of the SiNT-based transistors may be substantially improved by selective $p / n$ doping in the thinner side of the tube wall in the spirit of modulation doping. () 2007 American Institute of Physics.
\end{abstract} [DOI: $10.1063 / 1.2778756]$

Recently one-dimensional (1D) Si nanostructures such as tubes and wires have attracted much interest because of their significance in both fundamental research and potential technological applications. It was experimentally showed that crystalline Si nanowires ${ }^{1,2}$ (SiNWs) and $\mathrm{Si}$ nanotubes ${ }^{3}$ (SiNTs) are an ideal system to investigate quantum confinement effects leading to the useful size-dependent electronic and optical properties. Meanwhile, $a b$ initio calculations of Bai et al. predicted that the single-walled SiNTs, which have unique structural feature and are metallic, could be locally stable in vacuum at zero temperature. ${ }^{4}$ Variable electronic properties depending on the atomic configurations or sizes suggest that 1D Si nanostructures exhibit a range of device functions. Specially, they may be more readily integrated into conventional Si-based circuitry than other 1D nanomaterials. To date, considerable efforts have been made to improve the transport properties (e.g., high carrier mobility and transconductance) of (doped) crystalline SiNWs or SiNTs for the future electronic and photonic device applications. ${ }^{5,6}$ For the general doping technique used in crystalline SiNWs or SiNTs, however, significant backscattering induced by ionized impurities is present while increasing the carrier concentration, which then reduces the charge transmission for conducting channels. ${ }^{7}$ This motivates us to theoretically explore methods to circumvent the impurity scattering in these nanodevices.

The transport properties of (doped) SiNWs (Refs. 8 and 9) and SiNTs (Refs. 3 and 10) are to some extent sensitive to the quantum confinement effects. In the fabrication process, SiNTs with nonuniform wall thickness (nonuni- $\sigma$ ) are more frequently encountered as compared to ones with uniform wall thickness (uni- $\sigma$ ). Thus it would be very interesting to obtain insight into how the structural nonuniformity of crys-

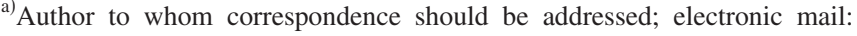
dwh@phys.tsinghua.edu.cn
}

talline SiNTs affects the electronic and optical properties because of the quantum confinement effects, and what is the dominating structural parameter of the nonuni- $\sigma$ tube. In this letter, we study quantum confinement effects of nonuni- $\sigma$ SiNTs using the first-principles method, and demonstrate the structural (i.e., uniformity and size) dependence of energy band gap, optical property, and wave functions of the valence band maximum (VBM) and conduction band minimum (CBM). Based on these findings, we suggest a possible experimental approach of modulation doping ${ }^{11}$ (i.e., the selective $p$ - $/ n$-type doping in the thinner sides) to achieve a relatively high carrier mobility by reducing impurity scattering of doped nonuni- $\sigma$ SiNTs.

Herein, crystalline SiNTs with the same external diameter of $13.37 \AA$ (see Fig. 1) are constructed from bulk crystalline $\mathrm{Si}$ along the low-index direction [100] (the $z$ axis). $\mathrm{H}$ termination is adopted to saturate all dangling bonds on both inner and outer surfaces. Our calculations are performed using the SIESTA code ${ }^{12,13}$ within the framework of density functional theory. The double-zeta polarized numerical atomic orbitals basis set is used. Periodic boundary condition is employed with at least $10 \AA$ separation between the two closest $\mathrm{H}$ atoms on neighboring tubes. The cutoff of grid integration of charge density is set to $150 \mathrm{Ry}$ and five Monkhorst-Pack $k$ points along the tube axis are used. Structural optimizations are performed using conjugate gradient method and deemed sufficiently converged when the residual forces on all ions are smaller than $0.02 \mathrm{eV} / \AA$.

The nonuni- $\sigma$ effects on the electronic structures of SiNTs are highlighted for two cases. (i) The atomic layers in the inner of the tube are removed in sequence, as shown in Figs. 1(a)-1(d). In this case, the band gap monotonically increases from 2.23 to $2.77 \mathrm{eV}$. (ii) The square hollow cavity is moved. As shown in Figs. 1(a) and 1(e) $-1(\mathrm{~g})$, the band gap decreases from 2.23 to $1.81 \mathrm{eV}$. As a representative, the projected density of states (PDOS) for the nonuni- $\sigma$ SiNT in 


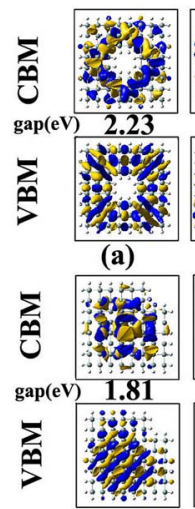

(g)

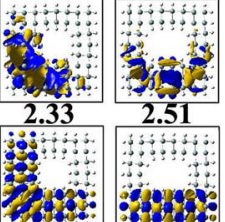

(b)
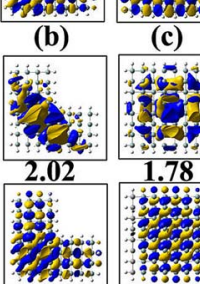

(h)
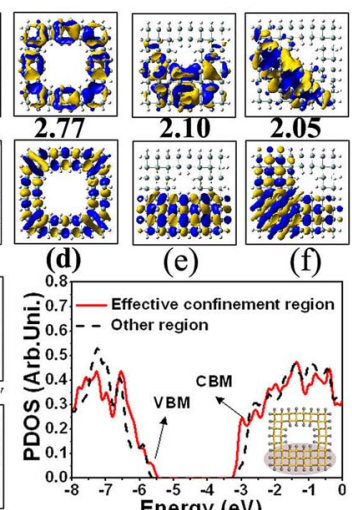

(j)
FIG. 1. (Color online) [(a)-(i)] Isosurface plots (in the $x y$ plane) of the wave functions of the valence band maximum (VBM) (bottom) and conduction band minimum (CBM) (top), together with corresponding band gaps, for SiNTs and SiNWs. Blue (dark gray) and yellow (light gray) regions correspond to positive and negative, respectively. The ball-and-stick structure models (viewed from the top) of corresponding SiNTs of SiNWs are plotted on the background with filled gray and light gray circles for $\mathrm{Si}$ and $\mathrm{H}$ atoms. All those isosurfaces correspond to an isovalue of $0.05 \mathrm{e} / \AA^{3}$. (j) Projected density of states of $\mathrm{Si}$ atoms in the effective confinement region (ECR) and other region. The CBM and VBM are labeled by arrows. The atomic structure is shown on the right as an inset and the ECR is indicated by gray background. (Filled yellow and gray circles for $\mathrm{Si}$ and $\mathrm{H}$ atoms.)

Fig. 1(c) is illustrated in Fig. 1(j). From the wave functions and PDOS plot, we can see that the electronic states near the Fermi level are mainly originated from the thicker sides of the wall. This feature is different from the case of uni- $\sigma$ SiNTs, where the wave functions of the VBM and CBM distribute uniformly on the four sides of the tube wall [see Figs. 1(a) and 1(d)]. In essence, the quantum transport of (doped) SiNTs is predominantly related to the electronic states near the VBM and CBM like as (doped) bulk $\mathrm{Si}^{10}$ Thus, the carriers (electrons and/or holes) in the nonuni- $\sigma$ SiNTs are mainly confined in the thicker side of the tube wall, which might be regarded as an "effective confinement region" (ECR). It can be confirmed that the band gap of nonuni- $\sigma$ SiNTs increases as the size of ECR reduces. In reality, the size of ECR indicates a substantial quantum confinement of 1D nanostructures. For example, the nonuni- $\sigma$ SiNT [Fig. 1(f)] and SiNW [Fig. 1(h)] with similar ECR exhibit similar wave functions of the VBM and CBM and have similar band gap. This quantum confinement effect of nonuni- $\sigma$ SiNTs can be generally understood by a "particle confined in a two-dimensional nonuni- $\sigma$ infinite potential well" model, and is insensitive to the shape of practical NTs (i.e., nonuni- $\sigma$ SiNTs along different orientations and with different atomic configurations will exhibit similar quantumsize confinement behavior). It should, however, be pointed out that the above effects induced by structural nonuniformity will be significantly reduced when the tube-wall thickness of SiNT far exceeds the Bohr exciton radius for bulk $\mathrm{Si}$ $(4.9 \mathrm{~nm}){ }^{14}$

In conventional semiconductors, the impurity scattering can be circumvented in heterorstructures such as GaAlAs/GaAs, by using the method of modulation doping. ${ }^{11}$ Taking into account the effects of nonuniform wall thickness on wave functions of the VBM and CBM for SiNTs as shown above, we can speculate on that a selective $p$-/ $n$-type doping in the thinner side of the tube wall will spatially separate the carriers (predominately distributing on the (a)

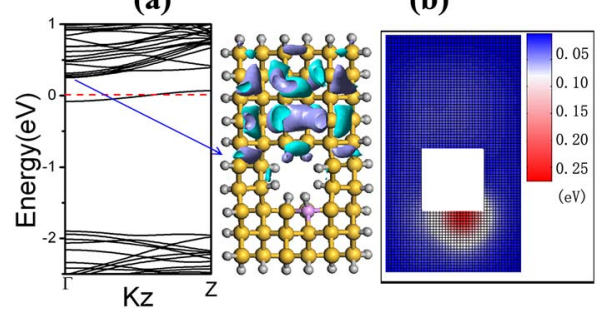

FIG. 2. (Color online) (a) Band structure for a P doped nanotube and the wave function of its lowest conduction band above the defect level together with its structure model. Filled yellow, light gray, and purple circles stand for $\mathrm{Si}, \mathrm{H}$, and $\mathrm{P}$ atoms. (b) Two-dimensional maps of on-site potential on transversal planes sectioning the tube.

thicker side) from the ionized impurities (doped in the thinner side), and hence the carriers will be weakly scattered by the ionized impurities in the transport. We can highlight such a result by investigating a phosphorus $(\mathrm{P})$ doped nonuni- $\sigma$ SiNT. As an example, a substitutional $\mathrm{P}$ atom is introduced in the thinner side of a SiNT, and then, the band structures and the on-site potential associated with $\mathrm{Si} s$ orbitals around the $\mathrm{P}$ impurity are calculated and given in Fig. 2. It can be seen that the doped P induces a defect level in the gap, which is localized around the $\mathrm{P}$ and surrounding $\mathrm{Si}$ atoms, while the lowest four conduction bands above the defect level still distribute mainly on the thicker side. This implies a minor interaction between the electrons in the conduction channel and the dopant. Meanwhile, a large potential change around $\mathrm{P}$ (in the thinner side) is observed, consistent with the P-doping case of SiNWs. ${ }^{7}$ However, the P dopant has much smaller effect on the potential in the thicker side. The above results may indicate less backscattering by the ionized impurity and higher conductance for the channels near the VBM and CBM. Moreover, our calculations of the formation energy show that for $\mathrm{P}$ doping, the thick side is more favorable in energy than the thin side by $0.07 \mathrm{eV}$, due to the internal stress difference at the doping sites in the two sides. Note that the model system studied is a very thin nanotube whose thin side has only two or three atomic layers. In the practical case, however, both the thick and thin sides of the tubes generally have more than tens of atomic layers. Consequently, one can expect that both sides will have similar stress distribution and almost the same formation energy for $\mathrm{P}$ dopant, while the nonuniform distribution feature of CBM and VBM is still preserved. This means that in synthesized nonuni- $\sigma$ SiNTs, it is possible to make the thin side selectively doped artificially for modulation doping. Especially, some efforts in technology have already been made to develop the area-selective doping method in nanoscale, e.g., selective dopant modulation during the growth of SiNWs. ${ }^{15}$ Even in the random doping case, the impurity scattering in SiNTs is believed to be weaker than that in SiNWs because of the existence of the thinner wall. So both electron ( $n$-type doping) and hole ( $p$-type doping) mobilities can be enhanced, and higher performance of nanodevices might be achieved. From the nonuniform distributions of the VBM and CBM caused by quantum confinement, we expect that this kind of modulation doping can be applied to other semiconductor nanostructures whose confined length is less than the corresponding Bohr exciton radius. Compared with the conventional modulation doping, our proposal does not depend on a heterostructure, and thereafter, the complication in AIP license or copyright; see http://apl.aip.org/apl/copyright.jsp 


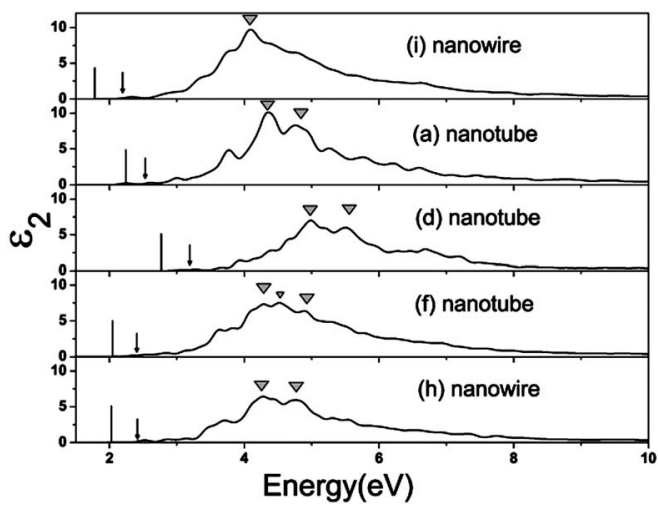

FIG. 3. Imaginary part of the dielectric function $\varepsilon_{2}$ polarized along the axial direction for SiNWs and SiNTs shown in Figs. 1(i), 1(a), 1(d), 1(f), and 1(h). The vertical lines and arrows indicate the band gaps and absorption edges, respectively. The absorption peaks are marked with inverted gray triangles.

transistor technology ${ }^{11}$ and the scattering by interface can be effectively avoided.

In order to obtain insight into the applications in optoelectronics or optics, we further study the nonuni- $\sigma$ effects on the optical properties of SiNTs by computing the imaginary part of the dielectric function $\varepsilon_{2}$ polarized along the tube axis, which is closely related to the optical absorption. The calculation is performed using an approach based on the dipole transition matrix elements between different eigenfunctions of the self-consistent Hamiltonian. ${ }^{16}$ Local field effect, exciton effect, and self-energy correction are not taken into account. Figure 3 shows the calculated $\varepsilon_{2}$ of several SiNWs and SiNTs in Fig. 1. Although all these nonuni- $\sigma$ [100] SiNTs studied here exhibit a direct band gap at $\Gamma$, the dipole transition matrix elements are still vanishingly small near the gap. Compared with the uni- $\sigma$ SiNW [Fig. 1(i)], those SiNTs with the same external diameter [Figs. 1(a), 1(d), and 1(f)] and the nonuni- $\sigma$ SiNW [Fig. 1(h)] exhibit a blueshift for both absorption peak and absorption edge. Different from the single absorption peak in the uni- $\sigma$ SiNW, two or three absorption peaks are present in these SiNTs and the nonuni- $\sigma$ SiNW due to different quantum confinement conditions (corresponding to the nonuniform wall thickness). For the uni- $\sigma$ SiNTs, the absorption peaks and edge also exhibit a substantial blue shift as the wall thickness decreases [see Figs. 1(a) and 1(d)]. While in the nonuni- $\sigma$ SiNT [Fig. 1(f)] with larger ECR than the uni- $\sigma$ SiNT [Fig. 1(a)], the absorption spectra shows a redshift, compatible with decreasing band gap. Owing to the similar ECR, the nonuni- $\sigma$ SiNW in Fig. 1(h) exhibits similar absorption spectra to the nonuni$\sigma$ SiNT in Fig. 1(f), except for a weak absorption peak.
In summary, the quantum confinement behavior, electronic, and optical properties of nonuni- $\sigma$ SiNTs are investigated through first-principles calculations. The distributions of wave functions of the VBM, CBM, and PDOS imply that the carriers (electrons and holes) may be mainly confined in the thicker sides of the nonuni- $\sigma$ SiNTs. The band gap decreases and the absorption spectra show a redshift with the enlargement of the ECR. Based on the effects of nonuniform wall thickness on wave functions of the VBM and CBM of the SiNT, a new modulation doping method, i.e., the selective $p$ - $/ n$-type doping in the thinner side, is proposed to improve the carrier mobility and transconductance of doped nonuni- $\sigma$ SiNTs. This approach is also valid for other semiconductor NTs used as nanoscale high-performance transistors, because it is expected that those properties related to nonuni- $\sigma$ are intrinsic for all semiconductor NTs.

This work was supported by the Ministry of Science and Technology of China (Grant Nos. 2006CB605105 and 2006CB0L0601) and the National Natural Science Foundation of China (Grant Nos. 10325415, 10404016, and 10674077). One of the authors (X.C.Z.) thanks the support from U.S. DOE (DE-FG02-04ER46164).

${ }^{1}$ J. Hu, T. W. Odom, and C. M. Lieber, Acc. Chem. Res. 32, 435 (1999). ${ }^{2}$ D. D. D. Ma, C. S. Lee, F. C. K. Au, S. Y. Tong, and S. T. Lee, Science 299, 1874 (2003).

${ }^{3}$ S. Y. Jeong, J. Y. Kim, D. Yang, B. N. Yoon, S. H. Choi, H. K. Kang, C. W. Yang, and Y. H. Lee, Adv. Mater. (Weinheim, Ger.) 15, 1172 (2003).

${ }^{4}$ J. Bai, X. C. Zeng, H. Tanaka, and J. Y. Zeng, Proc. Natl. Acad. Sci. U.S.A. 101, 2664 (2004).

${ }^{5}$ Y. Cui, Z. Zhong, D. Wang, W. U. Wang, and C. M. Lieber, Nano Lett. 3, 149 (2003).

${ }^{6}$ J. Xiang, W. Lu, Y. Hu, Y. Wu, H. Yan, and C. M. Lieber, Nature (London) 441, 489 (2006).

${ }^{7}$ M.-V. Fernádez-Serra, Ch. Adessi, and X. Blase, Nano Lett. 6, 2674 (2006).

${ }^{8}$ X. Duan, Y. Huang, Y. Cui, J. Wang, and C. M. Lieber, Nature (London) 409, 66 (2000).

${ }^{9}$ Y. Cui, Q. Q. Wei, H. K. Park, and C. M. Lieber, Science 293, 1289 (2001).

${ }^{10}$ B. Yan, G. Zhou, J. Wu, W. Duan, and B. Gu, Phys. Rev. B 73, 155432 (2006).

${ }^{11}$ S. Wang, Fundamentals of Semiconductor Theory and Device Physics (Prentice Hall, London, 1989), Chap. 11, p. 542.

${ }^{12}$ P. Ordejón, E. Artacho, and J. M. Soler, Phys. Rev. B 53, R10441 (1996).

${ }^{13}$ J. M. Soler, E. Artacho, J. D. Gale, A. Garcia, J. Junquera, P. Ordejón, and D. Sanchéz-Portal, J. Phys.: Condens. Matter 14, 2745 (2002).

${ }^{14}$ A. G. Cullis, L. T. Canham, and P. D. J. Calcott, J. Appl. Phys. 82, 909 (1997).

${ }^{15}$ C. Yang, Z. Zhong, and C. M. Lieber, Science 310, 1304 (2005).

${ }^{16}$ X. Zhao, C. M. Wei, L. Yang, and M. Y. Chou, Phys. Rev. Lett. 92, 236805 (2004) 